\title{
Effect of Tillage and Weed Control Methods in Maize (Zea mays) -Wheat (Triticum aestivum) Cropping System
}

\author{
R. R. Upasani ${ }^{1 *}$, Sheela Barla ${ }^{1}$ and A. N. Puran ${ }^{2}$
}

${ }^{1}$ Dept. of Agronomy, ${ }^{2}$ Dept. of Soil Science and Agricultural Chemistry, BAU, Ranchi, Jharkhand (834 006), India

\section{Corresponding Author}

R. R. Upasani

e-mail: upasani.ravikant@gmail.com
Article History

Article ID: 3 C0156

Received in $16^{\text {th }}$ September, 2017

Received in revised form $27^{\text {th }}$ November, 2017

Accepted in final form $2^{\text {nd }}$ December, 2017

\begin{abstract}
An experiment was conducted to find out the effect of tillage and weed control methods on weed dynamics and productivity of maize (Zea mays L.) -wheat (Triticum aestivum L.) cropping system. The treatments comprised of combinations of tillage sequences in main plots and weed control methods in sub plots. The results revealed that zero tillage in rainy and winter season is as good as conventional tillage method in reducing weed dry matter and producing maize and system yield, while, conventional tillage in rainy and winter seasons (CT-CT) and conventional tillage in rainy and zero tillage in winter (CT-ZT) reduced weed density as well as weed dry weight in wheat crop consequently, higher wheat grain yield was recorded compared to rest of the tillage sequences. Integrated weed management performed in maize and wheat (IWM-IWM) sequence recorded $51.78 \%$ and $61.96 \%$ higher maize grain yield and net return of maize, $18.40 \%$ and $17.23 \%$ higher wheat grain yield and net return of wheat, $31.78 \%$ and $34.96 \%$ higher system yield and net return respectively, compared to weedy check. Zero tillage with or without residue recorded improved soil $\mathrm{pH}$, organic carbon, $\mathrm{CO}_{2}$ and dehydrogenase activity. IWM in both the crops recorded higher yields, while significantly higher $\mathrm{B}: \mathrm{C}$ ratio was under recommended herbicide i.e. atrazine $1.0 \mathrm{~kg}$ ha-1 $\mathrm{pre}^{-}$ emergence in maize and 2,4-D $0.5 \mathrm{~kg} \mathrm{ha}^{-1}$ post emergence in wheat (RH-RH). IWM-IWM improved soil $\mathrm{CO}_{2}$ and dehydrogenase activity than $\mathrm{RH}-\mathrm{RH}$ sequences from their initial values.
\end{abstract}

Keywords: Productivity, tillage, weed dynamic

\section{Introduction}

Maize-wheat is the third most important cropping systems after rice-wheat and rice-rice that contributes about 3\% in the national food basket (Anonymous, 2013). Among different maize based cropping systems, maize-wheat ranks first having 1.8 mha area mainly concentrated in rainfed ecologies (Ghosh et al., 2015). The productivity of this system is declining after 1990 's. The low productivity of maize in India as compared to world productivity can be attributed to several limiting factors and all but the most important amongst these has been the poor weed management which poses a major threat to crop productivity. Weeds, being hardier in nature compete with maize plants for nutrients, water, sunlight and space during entire vegetative and early reproductive stages of maize; they transpire a lot of valuable conserved moisture and absorb large quantities of nutrients from the soil and their relative density plays a significant role in reducing the yield of crop (Kumar et al., 2015). Farmers plant wheat late because of multiple tillage operations (6-12). They believed that tillage was necessary for good germination, weed control, proper aeration and moisture conservation (Chauhan, 2000). Furthermore, intensive ploughing results to a decrease in soil organic matter due to acceleration of the oxidation and breakdown of organic matter and ultimately degradation of soil properties (Gathala et al., 2011). It also leads to compaction and eventually soil loss through wind and water erosion (Holland, 2004). Benefits of conservation tillage, especially zero-tillage (ZT) systems that leave crop residues on the soil surface are the stabilization of soil moisture and temperature, an improvement of aggregate stability and an increase in soil organic matter (Hajabbasi and Hemmat, 2000), higher water infiltration rates (Tullberg, 2010; Singh et al., 2011), and a reduction in weed population in some studies (Malik et al., 2004). However, negative effects of long-term ZT farming includes and increased weed competition (Mishra and Singh, 2012).The weeds are the major problem in the productivity of this system. They cause important yield losses 
worldwide with an average of $12.8 \%$ despite weed control applications and $29.2 \%$ in the case of no weed control (Oerke and Steiner, 1996) and, under very severe infestations the losses may go up to $80 \%$. The study of the weed dynamic in maize-wheat cropping system helps the researchers and farmers to formulate the strategies for the control of weeds. The present study was, therefore, undertaken with the objectives to evaluate the effect of tillage/planting management techniques and their interaction on weed dynamics and productivity of maize-wheat cropping system.

\section{Materials and Methods}

The present investigation was conducted in agronomical farm of Birsa Agricultural University, Ranchi during 2013-14, 201415 and 2015-16. The experimental soil was low in available nitrogen (167 kg ha-1) and medium in phosphorus (19 kg ha-1) and potash (187 kg ha-1) and the $\mathrm{pH}$ was 6.2. The treatments comprised of five different tillage sequences in main plots i.e. conventional tillage both in rainy and winter seasons (CT-CT), conventional tillage in rainy and zero tillage in winter seasons (CT-ZT), zero tillage both in rainy and winter seasons ( ZT-ZT), zero tillage in rainy and zero tillage along with crop residue in winter season ( $\mathrm{ZT}-\mathrm{ZT}+\mathrm{R}$ ) and zero tillage along with crop residue in rainy and winter seasons $(Z T+R-Z T+R)$. Weed control methods in sub plots were namely recommended herbicide i.e. atrazine $1.0 \mathrm{~kg} \mathrm{ha}^{-1}$ pre emergence in maize and 2,4-D 0.5 $\mathrm{kg} \mathrm{ha}^{-1}$ post emergence in wheat ( $\left.\mathrm{RH}-\mathrm{RH}\right)$, integrated weed management containing intercropping with black gram+pre emergence application of pendimethalin $1.0 \mathrm{~kg} \mathrm{ha}^{-1}$ followed by $(f b)$ manual weeding at 30 days after sowing (DAS) in maize and application of 2,4-D $0.5 \mathrm{~kg} \mathrm{ha}^{-1}$ post emergence in wheat $f b$ hand weeding at 40 DAS (IWM-IWM), and weedy check in rainy and winter seasons (WC-WC). The treatments were under fixed location during the years. Maize variety 'Suwan composite' and wheat 'K9107' were sown under irrigated conditions. The recommended dose of $\mathrm{N}, \mathrm{P}_{2} \mathrm{O}_{5}$ and $\mathrm{K}_{2} \mathrm{O}$ for maize and wheat was 120:60:40 $\mathrm{kg} \mathrm{ha}^{-1}$. Entire phosphorus and potassium were applied at the time of sowing to both the crops. In maize, nitrogen was applied in three equal splits at sowing, knee height and tasseling stages whereas in wheat, half nitrogen at the time of sowing and remaining nitrogen was applied in two equal splits at tillering and panicle initiation stages.

The observations on weed dynamics was focused on weed density and dry matter accumulation by weeds at 30 and 60 DAS. The weed densities were counted within quadrate of size $0.5 \times 0.5 \mathrm{~m}^{2}$ and were converted to per $\mathrm{m}^{2}$. The weed samples taken for recording observation on weed density at 30 and 60 DAS were sun dried to remove any excess moisture present on the surface of weeds then oven dried at $60^{\circ} \mathrm{C} \pm 5^{\circ} \mathrm{C}$. After complete oven drying the dry weight was recorded in $\mathrm{g} \mathrm{m}^{-2}$ on electronic balance. Data on weed density and dry matter of weeds were subjected to square root transformation i.e. $(X+0.5)$ prior to statistical analysis for test of significance. The treatment effects were compared using transformed means.

The analysis of variance method (Gomez and Gomez, 1984) was followed for statistical analysis of the various data.

\section{Results and Discussion}

\subsection{Study on weeds}

Maize: Tillage methods did not influence grassy, broad leaved and sedges as well as total weed density. The dominance of weeds under conventional and zero tillage may be because of different reasons. As under conventional tillage weed seeds which remained buried within soil might have transported up on the soil surface by soil disturbance while under zero tillage weed seeds which remained on soil surface germinated and grew resulting similar effect on weed density and dry matter at 30 and 60 DAS as that of conventional tillage. Matloob et al. (2015) have also observed grassy weeds were much higher under zero while broad-leaved weeds, especially Trianthemum portulacastrum dominated under continuous tillage system. Thus, the density and biomass of weeds was considerably similar under both the tillage systems (Table 1).

Among weed control methods, integrated weed management (IWM) performed in both the season recorded significantly reduced weed density as well as weed dry matter of all categories of weeds. Similar results were also observed by Bali et al. (2016). The decrease of grassy, broad leaved, sedges, and total weed density was to the tune of 53.33, $70.37,70.76$ and $63.23 \%$ compared to weedy check at 30 DAS, while at 60 DAS it was to the extent of 52.84, 57.27, 63.98 and $57.57 \%$, respectively compared to weedy check. Whereas, this treatment decreased weed dry matter to the tune of 52.24 and $64.89 \%$ compared to weedy check at 30 and 60 DAS respectively. Verma et al. (2015) have also suggested that for getting effective control of composite weed flora, a logical combination of several weed control methods is likely to prove the most effective approach.

Wheat: Continuous conventional tillage (CT-CT) similar to conventional tillage performed in rainy and zero tillage in winter season (CT-ZT) recorded significantly reduced weed density of all categories of weeds as well as dry matter of weeds at 30 and 60 DAS compared to other tillage sequences. Reduction in weed density and weed dry matter under conventional tillage in wheat crop has also been reported by Ramesh et al. (2014); Han et al. (2013) (Table 2).

Among weed control methods, integrated weed management (IWM) performed in both the season similar to recommended herbicide recorded significantly reduced weed density as well as weed dry matter of all categories of weeds. Total weed density at 30 and 60 DAS was decreased to the tune of 74.29 and $78.75 \%$ whereas, weed dry matter 13.07 and $74.03 \%$ 


\begin{tabular}{|c|c|c|c|c|c|c|c|c|c|c|}
\hline \multirow[t]{3}{*}{ Treatments } & \multicolumn{8}{|c|}{ Weed density (no. $\mathrm{m}^{-2}$ ) } & \multirow{2}{*}{\multicolumn{2}{|c|}{$\begin{array}{l}\text { Total weed dry } \\
\text { weight }\left(\mathrm{g} \mathrm{m}^{-2}\right)\end{array}$}} \\
\hline & \multicolumn{4}{|c|}{30 DAS } & \multicolumn{4}{|c|}{60 DAS } & & \\
\hline & Grassy & $\begin{array}{l}\text { Broad } \\
\text { leaved }\end{array}$ & Sedges & Total & Grassy & $\begin{array}{l}\text { Broad } \\
\text { leaved }\end{array}$ & Sedges & Total & 30 DAS & 60 DAS \\
\hline \multicolumn{11}{|c|}{ Tillage methods } \\
\hline $\mathrm{CT}-\mathrm{CT}$ & $\begin{array}{c}9.45 \\
(107.67)\end{array}$ & $\begin{array}{l}9.28 \\
(104)\end{array}$ & $\begin{array}{c}7.44 \\
(70.67)\end{array}$ & $\begin{array}{l}15.75 \\
(286)\end{array}$ & $\begin{array}{c}10.26 \\
(124.33)\end{array}$ & $\begin{array}{c}10.94 \\
(137.67)\end{array}$ & $\begin{array}{c}8.75 \\
(97.67)\end{array}$ & $\begin{array}{l}17.68 \\
(360)\end{array}$ & $\begin{array}{c}6.96 \\
(51.67)\end{array}$ & $\begin{array}{c}8.07 \\
(69.96)\end{array}$ \\
\hline $\mathrm{CT}-\mathrm{ZT}$ & $\begin{array}{c}8.54 \\
(86.67)\end{array}$ & $\begin{array}{c}9.01 \\
(98.67)\end{array}$ & $\begin{array}{c}7.36 \\
(69.67)\end{array}$ & $\begin{array}{l}14.98 \\
(259)\end{array}$ & $\begin{array}{l}10.44 \\
(128)\end{array}$ & $\begin{array}{l}10.30 \\
(121)\end{array}$ & $\begin{array}{c}8.69 \\
(94.33)\end{array}$ & $\begin{array}{l}17.34 \\
(343)\end{array}$ & $\begin{array}{c}7.02 \\
(51.63)\end{array}$ & $\begin{array}{c}8.56 \\
(78.7)\end{array}$ \\
\hline ZT-ZT & $\begin{array}{c}9.32 \\
(102.67)\end{array}$ & $\begin{array}{c}8.95 \\
(89.67)\end{array}$ & $\begin{array}{l}8.65 \\
(91)\end{array}$ & $\begin{array}{c}15.85 \\
(278.33)\end{array}$ & $\begin{array}{c}9.94 \\
(121.67)\end{array}$ & $\begin{array}{l}10.80 \\
(133)\end{array}$ & $\begin{array}{c}9.22 \\
(100.67)\end{array}$ & $\begin{array}{c}17.50 \\
(355.33)\end{array}$ & $\begin{array}{c}7.22 \\
(55.37)\end{array}$ & $\begin{array}{c}8.07 \\
(74.69)\end{array}$ \\
\hline $\mathrm{ZT}-\mathrm{ZT}+\mathrm{R}$ & $9.23(99)$ & $\begin{array}{c}9.86 \\
(110.67)\end{array}$ & $\begin{array}{c}9.29 \\
(108.33)\end{array}$ & $\begin{array}{l}16.52 \\
(314)\end{array}$ & $\begin{array}{l}10.67 \\
(133)\end{array}$ & $\begin{array}{l}10.35 \\
(126)\end{array}$ & $\begin{array}{c}9.14 \\
(103.67)\end{array}$ & $\begin{array}{c}17.58 \\
(362.67)\end{array}$ & $\begin{array}{c}7.06 \\
(53.23)\end{array}$ & $\begin{array}{c}8.26 \\
(77.37)\end{array}$ \\
\hline $\mathrm{ZT}+\mathrm{R}-\mathrm{ZT}+\mathrm{R}$ & $\begin{array}{c}8.85 \\
(94.33)\end{array}$ & $\begin{array}{l}9.97 \\
(114)\end{array}$ & $\begin{array}{l}9.09 \\
(105)\end{array}$ & $\begin{array}{l}15.95 \\
(297)\end{array}$ & $\begin{array}{c}11.42 \\
(152.33)\end{array}$ & $\begin{array}{c}10.59 \\
(127.67)\end{array}$ & $\begin{array}{l}9.23 \\
(104)\end{array}$ & $\begin{array}{c}18.30 \\
(384.67)\end{array}$ & $\begin{array}{c}7.04 \\
(52.8)\end{array}$ & $\begin{array}{c}8.61 \\
(85.03)\end{array}$ \\
\hline SEm \pm & 0.95 & 0.93 & 0.67 & 1.01 & 0.55 & 0.85 & 0.50 & 0.62 & 0.32 & 0.35 \\
\hline$C D(p=0.05)$ & NS & NS & NS & NS & NS & NS & NS & NS & NS & NS \\
\hline \multicolumn{11}{|l|}{ Weed control } \\
\hline $\mathrm{R} \mathrm{H}-\mathrm{RH}$ & $8.03(77)$ & $\begin{array}{c}7.81 \\
(70.33)\end{array}$ & $\begin{array}{c}7.04 \\
(60.67)\end{array}$ & $\begin{array}{c}13.21 \\
(195.33)\end{array}$ & $\begin{array}{l}8.52 \\
(82)\end{array}$ & $\begin{array}{c}8.98 \\
(90.67)\end{array}$ & $\begin{array}{c}7.66 \\
(72.33)\end{array}$ & $\begin{array}{l}14.73 \\
(245)\end{array}$ & $\begin{array}{c}5.96 \\
(36.21)\end{array}$ & $\begin{array}{c}7.06 \\
(22.02)\end{array}$ \\
\hline IWM-IWM & $7.63(67)$ & $\begin{array}{c}7.56 \\
(66.67)\end{array}$ & $\begin{array}{c}6.67 \\
(58.33)\end{array}$ & $\begin{array}{c}13.00 \\
(190.67)\end{array}$ & $\begin{array}{c}9.14 \\
(98.67)\end{array}$ & $\begin{array}{c}8.87 \\
(91.67)\end{array}$ & $\begin{array}{l}7.33 \\
(68)\end{array}$ & $\begin{array}{c}14.87 \\
(258.67)\end{array}$ & $\begin{array}{c}6.03 \\
(36.85)\end{array}$ & $\begin{array}{c}6.75 \\
(48.24)\end{array}$ \\
\hline WC-WC & $\begin{array}{l}11.58 \\
(150)\end{array}$ & $\begin{array}{c}12.88 \\
(173.33)\end{array}$ & $\begin{array}{c}11.39 \\
(147.67)\end{array}$ & $\begin{array}{c}21.22 \\
(474.33)\end{array}$ & $\begin{array}{l}13.97 \\
(215)\end{array}$ & $\begin{array}{c}13.95 \\
(204.67)\end{array}$ & $\begin{array}{c}12.02 \\
(160.33)\end{array}$ & $\begin{array}{c}23.43 \\
(580.33)\end{array}$ & $\begin{array}{c}9.18 \\
(85.75)\end{array}$ & $\begin{array}{c}11.13 \\
(103.37)\end{array}$ \\
\hline $\mathrm{SEm} \pm$ & 0.71 & 0.67 & 0.50 & 0.72 & 0.45 & 0.62 & 0.49 & 0.60 & 0.32 & 0.37 \\
\hline$C D(P=0.05)$ & 2.80 & 2.64 & 1.97 & 2.81 & 1.77 & 2.43 & 1.93 & 2.36 & 1.25 & 1.46 \\
\hline \multicolumn{11}{|l|}{ Interaction } \\
\hline SEm \pm & 0.87 & 0.93 & 0.90 & 0.87 & 0.72 & 0.93 & 0.72 & 0.74 & 0.39 & 0.40 \\
\hline $\mathrm{CD}(p=0.05)$ & NS & NS & NS & NS & NS & NS & NS & NS & 1.17 & NS \\
\hline
\end{tabular}

*Figures in parentheses are original values

Table 2: Effect of tillage and weed control methods on weed density and total dry weight of weed at different stages in wheat (Pooled data of 2013-14, 2014-15 and 2015-16)

\begin{tabular}{|c|c|c|c|c|c|c|c|c|}
\hline \multirow[t]{3}{*}{ Treatments } & & \multicolumn{5}{|c|}{ Weed density (no. $\mathrm{m}^{-2}$ ) } & \multicolumn{2}{|c|}{$\begin{array}{c}\text { Total weed dry weight } \\
\qquad\left(\mathrm{g} \mathrm{m}^{-2}\right)\end{array}$} \\
\hline & \multicolumn{3}{|c|}{30 DAS } & \multicolumn{3}{|c|}{60 DAS } & \multirow[t]{2}{*}{30 DAS } & \multirow[t]{2}{*}{60 DAS } \\
\hline & Grassy & $\begin{array}{l}\text { Broad } \\
\text { leaved }\end{array}$ & Total & Grassy & $\begin{array}{l}\text { Broad } \\
\text { leaved }\end{array}$ & Total & & \\
\hline \multicolumn{9}{|c|}{ Tillage methods } \\
\hline CT-CT & $\begin{array}{c}2.88 \\
(11.03)\end{array}$ & $\begin{array}{c}4.02 \\
(21.21)\end{array}$ & $\begin{array}{c}4.92 \\
(32.25)\end{array}$ & $\begin{array}{c}3.56 \\
(20.27)\end{array}$ & $\begin{array}{c}5.04 \\
(28.88)\end{array}$ & $\begin{array}{c}6.36 \\
(49.14)\end{array}$ & $\begin{array}{c}2.82 \\
(8.21)\end{array}$ & $\begin{array}{c}2.55 \\
(6.94)\end{array}$ \\
\hline CT-ZT & $\begin{array}{c}3.06 \\
(12.47)\end{array}$ & $\begin{array}{c}4.34 \\
(24.95)\end{array}$ & $\begin{array}{c}5.30 \\
(37.43)\end{array}$ & $\begin{array}{c}3.93 \\
(23.74)\end{array}$ & $\begin{array}{c}5.58 \\
(34.76)\end{array}$ & $\begin{array}{c}7.03 \\
(58.5)\end{array}$ & $\begin{array}{c}3.23 \\
(10.93)\end{array}$ & $\begin{array}{c}2.68 \\
(7.73)\end{array}$ \\
\hline
\end{tabular}




\begin{tabular}{|c|c|c|c|c|c|c|c|c|}
\hline \multirow[t]{3}{*}{ Treatments } & & \multicolumn{5}{|c|}{ Weed density (no. $\mathrm{m}^{-2}$ ) } & \multicolumn{2}{|c|}{$\begin{array}{l}\text { Total weed dry weight } \\
\qquad\left(\mathrm{g} \mathrm{m}^{-2}\right)\end{array}$} \\
\hline & \multicolumn{3}{|c|}{30 DAS } & \multicolumn{3}{|c|}{ 60D AS } & \multirow[t]{2}{*}{30 DAS } & \multirow[t]{2}{*}{60 DAS } \\
\hline & Grassy & $\begin{array}{l}\text { Broad } \\
\text { leaved }\end{array}$ & Total & Grassy & $\begin{array}{l}\text { Broad } \\
\text { leaved }\end{array}$ & Total & & \\
\hline ZT-ZT & $\begin{array}{c}4.01 \\
(20.69)\end{array}$ & $\begin{array}{c}5.03 \\
(32.85)\end{array}$ & $\begin{array}{c}6.42 \\
(53.54)\end{array}$ & $\begin{array}{c}4.68 \\
(29.96)\end{array}$ & $\begin{array}{c}7.01 \\
(55.01)\end{array}$ & $\begin{array}{c}8.62 \\
(84.97)\end{array}$ & $\begin{array}{c}3.58 \\
(14.11)\end{array}$ & $\begin{array}{c}3.30 \\
(12.71)\end{array}$ \\
\hline $\mathrm{ZT}-\mathrm{ZT}+\mathrm{R}$ & $\begin{array}{l}3.86 \\
(18.5)\end{array}$ & $\begin{array}{c}4.91 \\
(31.43)\end{array}$ & $\begin{array}{c}6.26 \\
(49.93)\end{array}$ & $\begin{array}{c}4.60 \\
(28.99)\end{array}$ & $\begin{array}{c}6.70 \\
(48.95)\end{array}$ & $\begin{array}{c}8.31 \\
(77.94)\end{array}$ & $\begin{array}{c}3.72 \\
(14.6)\end{array}$ & $\begin{array}{c}3.12 \\
(10.99)\end{array}$ \\
\hline $\mathrm{ZT}+\mathrm{R}-\mathrm{ZT}+\mathrm{R}$ & $\begin{array}{c}3.86 \\
(20.22)\end{array}$ & $\begin{array}{c}5.05 \\
(32.99)\end{array}$ & $\begin{array}{c}6.39 \\
(53.21)\end{array}$ & $\begin{array}{c}4.44 \\
(28.26)\end{array}$ & $\begin{array}{c}6.75 \\
(48.03)\end{array}$ & $\begin{array}{c}8.30 \\
(76.28)\end{array}$ & $\begin{array}{c}5.42 \\
(45.67)\end{array}$ & $\begin{array}{c}3.18 \\
(10.91)\end{array}$ \\
\hline SEm \pm & 0.20 & 0.18 & 0.16 & 0.18 & 0.36 & 0.34 & 0.28 & 0.17 \\
\hline$C D(p=0.05)$ & 0.64 & 0.59 & 0.52 & 0.6 & 1.18 & 1.09 & 0.92 & 0.56 \\
\hline \multicolumn{9}{|l|}{ Weed control } \\
\hline $\mathrm{R} \mathrm{H}-\mathrm{RH}$ & $\begin{array}{c}2.86 \\
(11.26)\end{array}$ & $\begin{array}{c}4.12 \\
(23.18)\end{array}$ & $\begin{array}{c}4.98 \\
(34.44)\end{array}$ & $\begin{array}{c}3.58 \\
(20.65)\end{array}$ & $\begin{array}{c}5.33 \\
(30.41)\end{array}$ & $\begin{array}{c}6.64 \\
(51.07)\end{array}$ & $\begin{array}{c}3.76 \\
(15.4)\end{array}$ & $\begin{array}{l}2.56 \\
(6.8)\end{array}$ \\
\hline IWM-IWM & $\begin{array}{l}2.46 \\
(7.6)\end{array}$ & $\begin{array}{c}3.94 \\
(20.27)\end{array}$ & $\begin{array}{c}4.59 \\
(27.86)\end{array}$ & $\begin{array}{c}3.33 \\
(17.97)\end{array}$ & $\begin{array}{c}4.69 \\
(23.22)\end{array}$ & $\begin{array}{c}5.93 \\
(41.19)\end{array}$ & $\begin{array}{c}3.52 \\
(12.58)\end{array}$ & $\begin{array}{c}2.31 \\
(5.09)\end{array}$ \\
\hline WC-WC & $\begin{array}{c}5.28 \\
(30.89)\end{array}$ & $\begin{array}{c}5.95 \\
(42.61)\end{array}$ & $\begin{array}{c}8.00 \\
(73.51)\end{array}$ & $\begin{array}{c}5.82 \\
(40.11)\end{array}$ & $\begin{array}{c}8.63 \\
(75.73)\end{array}$ & $\begin{array}{c}10.60 \\
(115.84)\end{array}$ & $\begin{array}{c}3.98 \\
(28.14)\end{array}$ & $\begin{array}{c}4.02 \\
(17.68)\end{array}$ \\
\hline $\mathrm{SEm} \pm$ & 0.11 & 0.2 & 0.08 & 0.08 & 0.1 & 0.09 & 0.01 & 0.01 \\
\hline$C D(p=0.05)$ & 0.42 & 0.8 & 0.3 & 0.3 & 0.38 & 0.36 & 0.03 & 0.04 \\
\hline \multicolumn{9}{|l|}{ Interaction } \\
\hline SEm \pm & 0.29 & 0.37 & 0.31 & 0.38 & 0.46 & 0.49 & 0.31 & 0.28 \\
\hline $\mathrm{CD}(p=0.05)$ & NS & NS & NS & NS & NS & NS & NS & NS \\
\hline
\end{tabular}

*Figures in parentheses are original values

compared to weedy check.

\subsection{Yield and economics}

Maize : Pooled dataof three years revealed that all the tillage methodsrecorded similarly in producing maize grain yield consequently net return and $\mathrm{B}: \mathrm{C}$ ratio. However, $\mathrm{B}: \mathrm{C}$ ratio was maximum under zero tillage compared to conventional tillage owing to saving in tillage operations thus reduced cost of cultivation. Similar was the findings of Marwat et al. (2011).

Integrated weed management (IWM) performed in maize recorded $51.78 \%$ and $61.96 \%$ higher grain yield and net return of maize compared to weedy check (WC-WC), due to crop received clear environment during critical period of crop weed competition and the condition was more favorable for its growth and development. Significantly lower yield under weedy check was also recorded by Pandit et al. (2016). However, maximum $\mathrm{B}: \mathrm{C}$ ratio was recorded under application of recommended herbicide i.e. atrazine $1.0 \mathrm{~kg} \mathrm{ha}^{-1}$ this may be due to application of herbicide is less labour intensive, resulting less costlier. The results are in agreement to the findings of Mahmoud et al. (2012) (Table 3).
Wheat: Pooled data revealedthatconventional tillage in rainy and winter seasons (CT-CT) and conventional tillage in rainy and zero tillage in winter (CT-ZT) recorded higher wheat grain yield compared to rest of the tillage sequences.

Among weed control methods integrated weed management performed in maize and wheat (IWM-IWM) sequence similar to recommended herbicide sequences $(\mathrm{RH}-\mathrm{RH})$ recorded 18.40 and $17.23 \%$ higher pooled grain yield and net return of wheat, compared to weedy check. Bali et al. (2016) emphasized judicious combinations of cultural and chemical methods of weed control as integrated weed management system is a desired practice that aims at reducing the dosage of herbicide to be applied with mechanical weeding, which will help in managing weeds in a best way for realizing to sustain and boost the production (Table 4).

\subsection{System productivity and profitability}

Different tillage sequence performed during rainy and winter seasons did not affect system yield as well as economics, although conventional tillage performed during rainy and winter seasons recorded maximum system pooled yield and 


\begin{tabular}{|c|c|c|c|c|c|c|c|c|c|c|c|c|c|}
\hline \multirow[t]{3}{*}{ Treatments } & \multicolumn{8}{|c|}{ Yield (kg ha $\left.{ }^{-1}\right)$} & \multirow{3}{*}{$\begin{array}{l}\text { Cost of } \\
\text { cultivation } \\
\left(₹ \mathrm{ha}^{-1}\right)\end{array}$} & \multicolumn{4}{|c|}{ Net return (₹ ha-1) } \\
\hline & \multicolumn{4}{|c|}{ Grain } & \multicolumn{4}{|c|}{ Straw } & & & & & \\
\hline & 2013 & 2014 & 2015 & Pooled & 2013 & 2014 & 2015 & Pooled & & 2013 & 2014 & 2015 & Pooled \\
\hline \multicolumn{14}{|c|}{ Tillage methods } \\
\hline CT-CT & 2415 & 1677 & 2714 & 2269 & 4076 & 2082 & 4464 & 3541 & 18990 & 25387 & 10329 & 30648 & 22121 \\
\hline CT-ZT & 2043 & 1872 & 2902 & 2272 & 3501 & 3438 & 4774 & 3904 & 18990 & 18657 & 15966 & 34088 & 22904 \\
\hline ZT-ZT & 1842 & 2694 & 2615 & 2384 & 3072 & 4048 & 3795 & 3638 & 14690 & 19084 & 33816 & 32125 & 28342 \\
\hline $\mathrm{ZT}-\mathrm{ZT}+\mathrm{R}$ & 2245 & 2839 & 1074 & 2053 & 3730 & 4362 & 4415 & 4169 & 14690 & 26445 & 36619 & 10250 & 24438 \\
\hline$Z T+R-Z T+R$ & 2104 & 3133 & 1506 & 2248 & 3539 & 5221 & 4588 & 4449 & 14690 & 23948 & 42747 & 17076 & 27924 \\
\hline SEm \pm & 102 & 251 & 378 & 244 & 184 & 144 & 434 & 303 & & 1506 & 3976 & 6220 & 3901 \\
\hline$C D(p=0.05)$ & 334 & 818 & 1232 & NS & 602 & 471 & NS & NS & & 4911 & 12964 & 20283 & NS \\
\hline \multicolumn{14}{|l|}{ Weed control } \\
\hline $\mathrm{R} \mathrm{H}-\mathrm{RH}$ & 2364 & 2672 & 2202 & 2413 & 4045 & 3996 & 4524 & 4188 & 14558 & 28992 & 33514 & 27520 & 30009 \\
\hline IWM-IWM & 2609 & 3098 & 3042 & 2916 & 4499 & 5232 & 5387 & 5039 & 20914 & 27219 & 36020 & 35490 & 32910 \\
\hline WC-WC & 1417 & 1559 & 1242 & 1406 & 2207 & 2262 & 3311 & 2593 & 13758 & 11911 & 14151 & 11494 & 12519 \\
\hline SEm \pm & 58 & 134 & 63 & 85 & 128 & 133 & 241 & 196 & & 745 & 2126 & 789 & 1220 \\
\hline $\mathrm{CD}(p=0.05)$ & 226 & 526 & 248 & 334 & 501 & 522 & 947 & 769 & & 2924 & 8346 & 3095 & 4789 \\
\hline \multicolumn{14}{|l|}{ Interaction } \\
\hline SEm \pm & 225 & 363 & 405 & 331 & 318 & 218 & 380 & 330 & & 3724 & 5691 & 6357 & 5257 \\
\hline $\mathrm{CD}(p=0.05)$ & NS & NS & 1214 & 992 & NS & 654 & 1138 & 989 & & NS & NS & 19057 & 15760 \\
\hline
\end{tabular}

\begin{tabular}{|c|c|c|c|c|}
\hline \multirow[t]{2}{*}{ Treatments } & \multicolumn{4}{|c|}{$\mathrm{B}: \mathrm{C}$ ratio } \\
\hline & 2013 & 2014 & 2015 & Pooled \\
\hline \multicolumn{5}{|c|}{ Tillage methods } \\
\hline CT-CT & 2.34 & 1.54 & 2.61 & 2.16 \\
\hline CT-ZT & 1.98 & 1.84 & 2.80 & 2.21 \\
\hline ZT-ZT & 2.30 & 3.30 & 3.19 & 2.93 \\
\hline $\mathrm{ZT}-\mathrm{ZT}+\mathrm{R}$ & 2.80 & 3.49 & 1.70 & 2.66 \\
\hline$Z T+R-Z T+R$ & 2.63 & 3.91 & 2.16 & 2.90 \\
\hline SEm \pm & 0.08 & 0.23 & 0.40 & 0.24 \\
\hline $\mathrm{CD}(p=0.05)$ & 0.27 & 0.77 & NS & NS \\
\hline \multicolumn{5}{|l|}{ Weed control } \\
\hline $\mathrm{R} \mathrm{H}-\mathrm{RH}$ & 2.99 & 3.30 & 2.89 & 3.06 \\
\hline IWM-IWM & 2.30 & 2.72 & 2.70 & 2.57 \\
\hline WC-WC & 1.87 & 2.03 & 1.84 & 1.91 \\
\hline SEm \pm & 0.03 & 0.12 & 0.11 & 0.09 \\
\hline$C D(P=0.05)$ & 0.12 & 0.47 & 0.45 & 0.34 \\
\hline \multicolumn{5}{|l|}{ Interaction } \\
\hline SEm \pm & 0.24 & 0.30 & 0.36 & 0.30 \\
\hline $\mathrm{CD}(p=0.05)$ & NS & 0.89 & 1.08 & 0.90 \\
\hline
\end{tabular}

$\mathrm{B}: \mathrm{C}$ ratio was calculated on the basis of gross return. Price of maize grain- ₹ $15 / \mathrm{kg}$, straw-₹ $2 / \mathrm{kg}$, wheat grain -₹ $13 /$ $\mathrm{kg}$, straw-₹3/kg

net return (Table 5).

Among weed control methods integrated weed management performed in maize and wheat (IWM-IWM) sequence similar to recommended herbicide sequences (RH-RH) recorded $31.78 \%$ and $34.96 \%$ higher system pooled yield and net return respectively, compared to weedy check. However, application of $\mathrm{RH}-\mathrm{RH}$ in maize and wheat recorded maximum pooled $\mathrm{B}: \mathrm{C}$ ratio that was 12.06 and $18.41 \%$ more compared to IWMIWM and WC-WC. Khaliq et al. (2013) also reported highest net benefits were associated with the use of label herbicide dose in all tillage practices. This may be due to low cost of cultivation under recommended herbicide as compared to integrated weed management.

\subsection{Soil properties}

Zero tillage in rainy and zero tillage along with crop residue in winter season (ZT-ZT+R) similar to $\mathrm{ZT}+\mathrm{R}-\mathrm{ZT}+\mathrm{R}$ recorded 5.63 $\mathrm{pH}$, which was $2.31 \%$ more than initial value and $3.37 \%$ more than conventional tillage sequences. ZT+R-ZT+R also recorded $3.16 \%$ and $14.37 \%$ higher organic carbon and $\mathrm{CO}_{2}$ respectively than initial values. $\mathrm{ZT}+\mathrm{R}-\mathrm{ZT}+\mathrm{R}$ similar to $\mathrm{ZT}-\mathrm{ZT}+\mathrm{R}$ and $\mathrm{CT}-\mathrm{ZT}$ recorded $7.76 \%$ more dehydrogenase than initial value and $26.06 \%$ more than conventional tillage sequences (CT-CT) which may be because of less soil disturbance under zero 


\begin{tabular}{|c|c|c|c|c|c|c|c|c|c|c|c|c|c|}
\hline \multirow[t]{3}{*}{ Treatments } & \multicolumn{8}{|c|}{ Yield $\left(\mathrm{kg} \mathrm{ha}^{-1}\right)$} & \multirow{3}{*}{$\begin{array}{c}\text { Cost of } \\
\text { cultiva- } \\
\text { tion (₹ } \\
\left.\text { ha }^{-1}\right)\end{array}$} & \multirow{2}{*}{\multicolumn{4}{|c|}{ Net return (' ha ${ }^{-1}$ ) }} \\
\hline & \multicolumn{4}{|c|}{ Grain } & \multicolumn{4}{|c|}{ Straw } & & & & & \\
\hline & 2013 & 2014 & 2015 & Pooled & 2013 & 2014 & 2015 & Pooled & & 2013 & 2014 & 2015 & Pooled \\
\hline \multicolumn{14}{|c|}{ Tillage methods } \\
\hline CT-CT & 4.54 & 4.48 & 3.86 & 4.29 & 5.63 & 7.20 & 7.12 & 6.65 & 24204 & 51706 & 55636 & 47601 & 51648 \\
\hline CT-ZT & 4.08 & 4.06 & 3.64 & 3.93 & 5.61 & 6.97 & 6.87 & 6.48 & 22454 & 47416 & 51236 & 45716 & 48123 \\
\hline ZT-ZT & 3.88 & 3.74 & 3.09 & 3.62 & 5.35 & 6.75 & 6.61 & 6.24 & 22454 & 44036 & 46416 & 37797 & 42750 \\
\hline$Z T-Z T+R$ & 3.96 & 3.94 & 3.20 & 3.70 & 5.19 & 6.69 & 7.31 & 6.40 & 22454 & 44596 & 48836 & 41299 & 44910 \\
\hline $\mathrm{ZT}+\mathrm{R}-\mathrm{ZT}+\mathrm{R}$ & 4.00 & 3.97 & 2.94 & 3.58 & 5.19 & 6.57 & 7.82 & 6.53 & 22454 & 45116 & 48866 & 39459 & 44480 \\
\hline SEm \pm & 0.30 & 0.19 & 0.09 & 0.18 & 0.28 & 0.39 & 0.24 & 0.30 & & 4506 & 3514 & 1424 & 3148 \\
\hline$C D(p=0.05)$ & NS & 0.62 & 0.29 & 0.58 & NS & NS & NS & NS & & NS & 11458 & 4643 & NS \\
\hline \multicolumn{14}{|l|}{ Weed control } \\
\hline $\mathrm{R} \mathrm{H}-\mathrm{RH}$ & 4.15 & 4.13 & 3.63 & 3.97 & 5.52 & 7.07 & 7.63 & 6.74 & 22535 & 47975 & 52365 & 47573 & 49304 \\
\hline IWM-IWM & 4.40 & 4.38 & 3.61 & 4.13 & 5.67 & 7.30 & 7.89 & 6.95 & 25382 & 48828 & 53458 & 45270 & 49185 \\
\hline WC-WC & 3.73 & 3.61 & 2.78 & 3.37 & 5.00 & 6.14 & 5.92 & 5.69 & 20495 & 42995 & 44855 & 34280 & 40710 \\
\hline SEm \pm & 0.11 & 0.06 & 0.10 & 0.12 & 0.24 & 0.22 & 0.37 & 0.28 & & 1646 & 468 & 1103 & 1072 \\
\hline $\mathrm{CD}(p=0.05)$ & 0.43 & 0.23 & 0.39 & 0.48 & NS & 0.88 & 1.44 & 1.10 & & NS & 1836 & 4330 & 4208 \\
\hline \multicolumn{14}{|l|}{ Interaction } \\
\hline SEm \pm & 0.50 & 0.53 & 0.21 & 0.31 & 0.36 & 0.55 & 0.54 & 0.48 & & 7038 & 7479 & 4359 & 6292 \\
\hline $\mathrm{CD}(p=0.05)$ & NS & NS & NS & NS & NS & NS & NS & NS & & NS & NS & 13067 & NS \\
\hline
\end{tabular}

Table 4: Contiue

\begin{tabular}{|c|c|c|c|c|}
\hline \multirow{2}{*}{ Treatments } & \multicolumn{4}{|c|}{$\mathrm{B}: \mathrm{C}$ ratio } \\
\hline & 2013 & 2014 & 2015 & Pooled \\
\hline \multicolumn{5}{|c|}{ Tillage methods } \\
\hline CT-CT & 3.14 & 3.30 & 2.98 & 3.14 \\
\hline CT-ZT & 3.11 & 3.28 & 3.07 & 3.15 \\
\hline ZT-ZT & 2.96 & 3.07 & 2.69 & 2.91 \\
\hline$Z T-Z T+R$ & 2.99 & 3.17 & 2.86 & 3.01 \\
\hline $\mathrm{ZT}+\mathrm{R}-\mathrm{ZT}+\mathrm{R}$ & 3.01 & 3.18 & 2.80 & 3.00 \\
\hline SEm \pm & 0.20 & 0.16 & 0.20 & 0.48 \\
\hline $\mathrm{CD}(p=0.05)$ & NS & NS & 0.65 & NS \\
\hline \multicolumn{5}{|l|}{ Weed control } \\
\hline RH --- RH & 3.13 & 3.32 & 3.11 & 3.19 \\
\hline IWM - IWM & 2.92 & 3.11 & 2.78 & 2.94 \\
\hline$W C-W C$ & 3.10 & 3.19 & 2.75 & 3.01 \\
\hline SEm \pm & 0.08 & 0.03 & 0.04 & 0.05 \\
\hline$C D(P=0.05)$ & NS & 0.10 & 0.16 & 0.2 \\
\hline \multicolumn{5}{|l|}{ Interaction } \\
\hline SEm \pm & 0.31 & 0.33 & 1.21 & 0.62 \\
\hline$C D(p=0.05)$ & NS & NS & 3.63 & 1.86 \\
\hline
\end{tabular}

$\mathrm{B}: \mathrm{C}$ ratio was calculated on the basis of gross return. Price of maize grain- ₹ 15/kg, straw-₹ 2/kg, wheat grain -₹ 13 / $\mathrm{kg}$, straw-₹ $3 / \mathrm{kg}$

tillage bringing up favorable soil environment for soil flora and fauna to survive. This also brought up similar effects under IWM-IWM and WC-WC in enhancing soil $\mathrm{CO}_{2}$ and dehydrogenase than initial value. According to Mutiu et al. (2015) zero or minimum tillage is beneficial to soil physical improvement as process of soil physical degradation normally sets in immediately after conventional tillage. Research reports indicate that conservation tillage, particularly under minimum tillage, is better than conventional tillage in terms of soil chemical improvement. All available reports are in agreement that soils under conservation tillage are more favoured than conventional tillage in terms of soil fauna activities and biological properties improvement. Małecka et al. (2012) have also found accumulation of organic carbon at the soil surface under reduced tillage and no tillage. The concentration of organic $\mathrm{C}$ in reduced tillage and particularly in no tillage, had increased significantly in the top layer (0-5 $\mathrm{cm}$ ), by $18.3 \%$ and $26.1 \%$, respectively, in comparison with CT Among weed control methods, WC-WC and IWM-IWM performed similar in enhancing soil $\mathrm{CO}_{2}$ and dehydrogenase. Weedy check recorded $4.53 \%, 5.34 \%$ and $13.27 \%$ higher $\mathrm{CO}_{2}$ and $0.79 \%, 0.31 \%$ and $7.56 \%$ higher dehydrogenase than initial value, IWM-IWM and $\mathrm{RH}-\mathrm{RH}$, respectively (Table 5). 


\begin{tabular}{|c|c|c|c|c|c|c|c|c|c|c|c|c|c|}
\hline \multirow[t]{2}{*}{ Treatments } & \multicolumn{4}{|c|}{ System yield $\left(\mathrm{t} \mathrm{ha}{ }^{-1}\right)$} & \multirow{2}{*}{$\begin{array}{c}\text { Cost of } \\
\text { cultiva- } \\
\text { tion (₹ } \\
\text { ha) }\end{array}$} & \multicolumn{4}{|c|}{ Net return (₹ ha) } & \multicolumn{4}{|c|}{$\mathrm{B}: \mathrm{C}$ ratio } \\
\hline & $\begin{array}{c}2013- \\
14\end{array}$ & $\begin{array}{c}2014- \\
15\end{array}$ & $\begin{array}{c}2015- \\
16\end{array}$ & Pooled & & $\begin{array}{c}2013- \\
14\end{array}$ & $\begin{array}{c}2014- \\
15\end{array}$ & $\begin{array}{c}2015- \\
16\end{array}$ & Pooled & $\begin{array}{c}2013- \\
14\end{array}$ & $\begin{array}{c}2014- \\
15\end{array}$ & $\begin{array}{c}2015- \\
16\end{array}$ & Pooled \\
\hline \multicolumn{14}{|c|}{ Tillage methods } \\
\hline CT-CT & 8.02 & 7.28 & 8.08 & 7.79 & 43194 & 77096 & 66012 & 77969 & 73692 & 2.77 & 2.50 & 2.81 & 2.69 \\
\hline $\mathrm{CT}-\mathrm{ZT}$ & 7.17 & 7.24 & 8.06 & 7.49 & 41444 & 66123 & 67182 & 79522 & 70942 & 2.61 & 2.60 & 2.92 & 2.71 \\
\hline ZT-ZT & 6.68 & 7.82 & 7.12 & 7.21 & 37144 & 63053 & 80161 & 69638 & 70951 & 2.70 & 3.15 & 2.87 & 2.91 \\
\hline $\mathrm{ZT}-\mathrm{ZT}+\mathrm{R}$ & 7.21 & 8.17 & 5.89 & 7.09 & 37144 & 71029 & 85390 & 51261 & 69227 & 2.92 & 3.33 & 2.38 & 2.88 \\
\hline $\mathrm{ZT}+\mathrm{R}-\mathrm{ZT}+\mathrm{R}$ & 7.08 & 8.75 & 6.23 & 7.35 & 37144 & 69076 & 94086 & 56252 & 73138 & 2.88 & 3.57 & 2.51 & 2.99 \\
\hline SEm \pm & 0.25 & 0.39 & 0.35 & 0.33 & & 3695 & 5793 & 5245 & 4911 & 0.10 & 0.15 & 0.09 & 0.11 \\
\hline$C D(p=0.05)$ & 0.82 & 1.27 & 1.14 & NS & & 12049 & 18889 & 17103 & NS & NS & 0.48 & 0.29 & NS \\
\hline \multicolumn{14}{|l|}{ Weed control } \\
\hline $\mathrm{R} \mathrm{H}-\mathrm{RH}$ & 7.60 & 8.19 & 7.48 & 7.76 & 37093 & 76956 & 85810 & 75098 & 79288 & 3.08 & 3.35 & 3.02 & 3.15 \\
\hline IWM-IWM & 8.16 & 9.05 & 8.47 & 8.56 & 46296 & 76029 & 89480 & 80762 & 82090 & 2.64 & 2.94 & 2.74 & 2.77 \\
\hline WC-WC & 5.94 & 6.31 & 5.28 & 5.84 & 34252 & 54841 & 60409 & 44927 & 53392 & 2.61 & 2.80 & 2.31 & 2.57 \\
\hline SEm \pm & 0.13 & 0.17 & 0.15 & 0.15 & & 1950 & 2504 & 2325 & 2260 & 0.05 & 0.06 & 0.06 & 0.06 \\
\hline $\mathrm{CD}(p=0.05)$ & 0.51 & 0.66 & 0.59 & 0.59 & & 7653 & 9829 & 9126 & 8871 & 0.19 & 0.24 & 0.24 & 0.24 \\
\hline \multicolumn{14}{|l|}{ Interaction } \\
\hline SEm \pm & 0.56 & 0.59 & 0.44 & 0.53 & & 8345 & 8904 & 6580 & 7943 & 0.22 & 0.22 & 0.12 & 0.19 \\
\hline$C D(p=0.05)$ & NS & NS & 1.32 & NS & & & & 19725 & 23811 & NS & NS & 0.36 & 0.57 \\
\hline
\end{tabular}

B:C ratio was calculated on the basis of gross return. Price of maize grain- ₹ 15/kg, straw-₹ $2 / \mathrm{kg}$, wheat grain $-₹ 13 / \mathrm{kg}$, straw-₹ $3 / \mathrm{kg}$

\begin{tabular}{|c|c|c|c|c|c|}
\hline Treatments & $\mathrm{pH}$ & OC g kg ${ }^{-1}$ soil & $\begin{array}{c}\mathrm{CO}_{2} \mathrm{mg}_{\mathrm{day}^{-1}} 100 \mathrm{~g}^{-1} \text { soil } \\
\end{array}$ & $\begin{array}{c}\text { Dehydrogenase } \\
\text { ( } \mu \mathrm{g} \mathrm{TPF} \mathrm{hr} \mathrm{g}^{-1} \mathrm{~g}^{-1} \text { soil) }\end{array}$ & $\begin{array}{l}\text { Azato-bacter } \\
\mathrm{cfu}(\mathrm{X103)}\end{array}$ \\
\hline \multicolumn{6}{|c|}{ Tillage Methods } \\
\hline $\mathrm{CT}-\mathrm{CT}$ & 5.44 & 4.48 & 5.55 & 5.05 & 2.56 \\
\hline $\mathrm{CT}-\mathrm{ZT}$ & 5.44 & 4.59 & 5.25 & 6.19 & 3.11 \\
\hline ZT-ZT & 5.52 & 4.86 & 5.37 & 6.07 & 2.89 \\
\hline $\mathrm{ZT}-\mathrm{ZT}+\mathrm{R}$ & 5.63 & 4.83 & 5.93 & 6.78 & 3.22 \\
\hline $\mathrm{ZT}+\mathrm{R}-\mathrm{ZT}+\mathrm{R}$ & 5.62 & 5.06 & 6.89 & 6.83 & 3.89 \\
\hline SEm \pm & 0.03 & 0.06 & 0.24 & 0.19 & 0.27 \\
\hline$C D(p=0.05)$ & 0.09 & 0.21 & 0.80 & 0.64 & NS \\
\hline \multicolumn{6}{|l|}{ Weed control } \\
\hline RH --- RH & 5.54 & 4.71 & 5.36 & 5.87 & 2.80 \\
\hline IWM - IWM & 5.47 & 4.79 & 5.85 & 6.33 & 3.20 \\
\hline$W C-W C$ & 5.57 & 4.79 & 6.18 & 6.35 & 3.40 \\
\hline SEm \pm & 0.04 & 0.03 & 0.09 & 0.10 & 0.29 \\
\hline$C D(P=0.05)$ & NS & NS & 0.34 & 0.38 & NS \\
\hline
\end{tabular}


Upasani et al., 2017

\begin{tabular}{|c|c|c|c|c|c|}
\hline Treatments & $\mathrm{pH}$ & $\mathrm{OC} \mathrm{g} \mathrm{kg}^{-1}$ soil & $\begin{array}{c}\mathrm{CO} 2 \mathrm{mg} 100 \mathrm{~g}^{-1} \text { soil } \\
\text { day }^{-1}\end{array}$ & $\begin{array}{c}\text { Dehydrogenase } \\
\text { ( } \mu \mathrm{g} \text { TPF hr-1 } \mathrm{g}^{-1} \text { soil) }\end{array}$ & $\begin{array}{l}\text { Azato-bacter } \\
\mathrm{cfu}(\mathrm{X103)}\end{array}$ \\
\hline \multicolumn{6}{|l|}{ Interaction } \\
\hline SEm \pm & 0.06 & 0.09 & 0.44 & 0.61 & 0.35 \\
\hline $\mathrm{CD}(p=0.05)$ & NS & NS & NS & NS & NS \\
\hline CV\% & 1.94 & 3.14 & 13.18 & 17.17 & 19.25 \\
\hline Initial value & 5.50 & 4.90 & 5.90 & 6.30 & 3.00 \\
\hline
\end{tabular}

\section{Conclusion}

Continuous zero tillage sequences with or without residue in maize wheat cropping system is more beneficial than conventional tillage. Application of recommended herbicide in maize and wheat is more profitable to farmers followed by integrated weed management sequences method for controlling weeds and attaining higher yield.

\section{References}

Anonymous, 2013. Agricultural Statistics at a Glance . Department of Agriculture and Cooperation, Ministry of Agriculture, Government of India, New Delhi, Available at www.dacnet.nic.in.

Bali, A., Bazaya, B.R., Chand, L., Swami, S., 2016. Weed management in soybean (Glycine max L.). The Bioscan 11(1), 255-257.

Chauhan, D.S., 2000. Wheat cultivation after rice a paradigm shift in tillage technology. Indian Farming 50(6), 21-22.

Gathala, M.K., Ladha, J.K., Kumar, V., Saharawat, Y.S., Kumar, V., Sharma, P.K, Sharma, S., Pathak, H., 2011. Tillage and crop establishment affects sustainability of South Asian Rice-wheat system. Agronomy Journal 103, 961-971.

Ghosh, B.N., Dogra, P., Sharma, N.K., Bhattacharya, R., Mishra, P.K., 2015. Conservation agriculture impact for soil conservation in maize-wheat cropping system in the Indian sub-Himalayas. International Soil and Water Conservation Research 3(2), 112-118.

Gomez, K.A., Gomez, A.A., 1984. Statistical procedures for agricultural research, (2 Edn.), John wiley and sons, NewYork, 680.

Hajabbasi, M.A., Hemmat, A., 2000. Tillage impacts on aggregate stability and crop productivity in a clay-loam soil in central Iran. Soil and Tillage Research 56, 205-212.

Han, H., Ning, T., Li, Z., 2013. Effects of tillage and weed management on the vertical distribution of microclimate and grain yield in a winter wheat field. Plant Soil Environment 59(5), 201-207.

Holland, J.M., 2004. The environmental consequences of adopting conservation tillage in Europe: reviewing the evidence. Agriculture, Ecosystems and Environment 103, 1-25.

Khaliq, A., Shakeel, M., Matloob, A.,, Hussain, S., Tanveer, A.,
Murtaza, G., 2013. Influence of Tillage and Weed Control Practices on Growth and Yield of Wheat. Philippine Journal of Crop Science 38(3), 54-62.

Kumar, A., Kumar, J., Puniya, R., Mahajan, A., Sharma, N., Stanzen, L., 2015. Weed management in maize-based cropping system. Indian Journal of Weed Science 47(3), 254-266.

Mahmoud, G., Nejad, R., Javad, S., Davoodi, M., Morteza, A., Houshang, B., Eskandar, Z., Alamisaeid, K., 2012. Effect of tillage, fertilization and weed control methods on corn yield in Khuzestan province. Scientific Research and Essays 7(43), 3727-3736.

Malik, R.K., Gupta, R.K., Singh, C.M., Brar, S.S., Singh, S.S., Sinha, R.K., Singh, A.K., Singh, R., Naresh, R.K., Singh, K.P., Thakur, T.C., 2004. Accelerating the adoption of resource conservation technologies for farm level impact on sustainability of rice-wheat system of the Indo-Gangetic plains. NATP Progress Report, CCSHAU, Haryana, India.

Marwat, K.B., Khan, M.A., Hashim, S., Nawab, K., Khattak, A.M., 2011. Integrated weed management in wheat. Pakistan Journal of Botany 43, 625-633.

Matloob, A., Abdul, K., Asif, T., Saddam, H., Farhena, A., Bhagirath, S.C., 2015. Weed dynamics as influenced by tillage system, sowing time and weed competition duration in dry-seeded rice. Crop Protection 71, 25-38.

Mishra, J.S., Singh, V.P., 2012. Tillage and weed control effects on productivity of a dry seeded rice-wheat system on a Vertisol in Central India. Soil and Tillage Research 123, 11-20.

Małecka, I., Andrzej, B., Zuzanna, S., Ttomasz, D., 2012. The effect of various long-term tillage systems on soil properties and spring barley yield. Turkish Journal of Agriculture and Forestry 36, 217-226.

Mutiu, A., Busari, S., Singh, Kukal, A.K., Bhatt, R., Dulazi, A.A., 2015. Conservation tillage impacts on soil, crop and the environment. International Soil and Water Conservation Research 3(2), 119-129.

Oerke, E.C., Steiner, U., 1996. Abschätzung der ErtragsverlusteimMaisanbau. In: Ertragsverluste und Pflanzenschutz-Die Anbausituationfür die wirtschaftlichwichtigsten Kulturpflanzen. German Phytomedical Society Series, Band: 6, 63-79, Eugen 
Ulmer Verlag, Stuttgart.

Pandit, S., Rathod, D.B.M., Patil, D.H., 2016. Integrated weed management in pigeonpea gement in pigeonpea (Cajanus cajan L.) under rainfed conditions of Karnataka. The Bioscan 11(1), 583-588.

Ramesh, S., Rana, S., Kumar, S., 2014. Weed dynamics and productivity of maize-wheat cropping system as influenced by tillage/planting techniques. Environment and Technology 3(3), 1059-1070.

Singh, Y., Singh, V.P., Singh, G., Yadav, D.S., Sinha, R.K.P., Johnson, D.E., Mortimer, A.M., 2011. The implications of land preparation, crop establishment method and weed management on rice yield variation in the rice-wheat system in the Indo-gangetic plains. Field Crops Research $121,64-74$.

Tullberg, J., 2010.Tillage, traffic and sustainability-a challenge for ISTRO. Soil and Tillage Research 111 (1), 26-32.

Verma, S.K., Singh, S.B., Meena, R.N., Prasad, S.K., Meena, R.S., Gaurav, 2015. A review of weed management in india: the need of new directions for sustainable agriculture. The Bioscan 10(1), 253-263. 